\title{
A Helm model for microRNA regulation in cell fate decision and conversion
}

\author{
XIE ShuJuan, ZHANG Yin, QU LiangHu* \& XU Hui* \\ Key Laboratory of Gene Engineering of Ministry of Education, State Key Laboratory for Biocontrol, Sun Yat-sen University, \\ Guangzhou 510275, China
}

Received August 2, 2013; accepted August 24, 2013; published online September 3, 2013

\begin{abstract}
microRNAs (miRNAs) constitute a unique class of endogenous small non-coding RNAs that regulate gene expression post-transcriptionally. Studies over the past decade have uncovered a recurring paradigm in which miRNAs are key regulators of cellular behavior under various physiological and pathological conditions. Most surprising is the recent observation that miRNAs have emerged as competent players in somatic cell reprogramming, suggesting an especially significant role for these small RNAs in cell fate settings. Here, we discuss the possible mechanisms underlying miRNA-mediated cell programming (i.e., the development and differentiation of embryonic stem cells) and reprogramming (i.e., turning somatic cells into pluripotent stem cells or other lineages), and provide a "Helm" model of miRNAs in cell fate decision and conversion.
\end{abstract}

miRNA(s), cell fate, development, differentiation, embryonic stem cells, reprogramming, iPS, transdifferentiation

Citation: Xie S J, Zhang Y, Qu L H, et al. A Helm model for microRNA regulation in cell fate decision and conversion. Sci China Life Sci, 2013, 56: 897-906, doi: $10.1007 / \mathrm{s} 11427-013-4547-4$

The discovery of microRNA (miRNA) has opened up a new view of biosystems because it revealed an unexpected, but huge, RNA world hidden in the genome [1]. Unlike messenger RNAs (mRNAs), ribosomal RNAs (rRNAs), or transfer RNAs (tRNAs) that directly take part in protein biosynthesis, miRNAs [2] and other small RNAs [3], or large non-coding RNAs which were found later [4], primarily function as regulatory molecules and modulate the expression of genes at multiple levels, directly or indirectly. Today, it is widely recognized that a complicated "RNA regulatory network" controls many, if not all, gene activities within a cell, including DNA and histone modification, structural dynamics of chromosome, gene transcription, RNA processing, transport, protein synthesis, and degradation [5].

One major characteristic of miRNAs that makes them

*Corresponding author (email: xuhui27@mail.sysu.edu.cn; 1ssqlh@mail.sysu.edu.cn) ideal regulators of cell activities, like transcription factors, is the ability of a single miRNA to directly control the expression of hundreds of genes to execute entire cellular or organ programs [6]. With miRNA roles identified in developmental timing, cell death, cell proliferation, hematopoiesis, and neuronal patterning [7], as well as in various human diseases, including cancers, cardiovascular diseases, neurodegeneration, diabetes, and viral infection [8], evidence is mounting that animal miRNAs are more numerous, and that their regulatory impact is more pervasive, than was previously suspected. Particularly, the most stunning example is that miRNAs help to orchestrate developmental events throughout embryonic development by regulating the temporal transitions in gene expression associated with cell fate progression and differentiation, which is well-characterized in lower animals such as Caenorhabditis elegans [9].

Recent years have seen rapid advances in the field of cell reprogramming, specifically in the conversion of kinds of 
somatic cells into pluripotent stem cells or into other lineages by defined factors $[10,11]$. Most striking is the observation that the ability to drive these transitions is not limited to transcription factors, but that they can be achieved by miRNAs as well $[12,13]$. Understanding the mechanisms of miRNA-based reprogramming may provide further insights into fundamental questions regarding cell plasticity, cell identity and cell fate decisions [14]. Here, rather than attempting to present a comprehensive survey of the numerous studies that have linked miRNAs to cell programming and reprogramming, we primarily focus on the unique modality by which miRNAs specify cell fate and discuss the influence of different miRNA families on the establishment and maintenance of cell identity.

\section{1 miRNAs promote programs that define the fate and characteristics of developing cells}

Development is an extremely complex and highly programmed process, by which a single zygote undergoes growth, fate determination, differentiation, morphogenesis, and finally forms an intact organism. From the day it was discovered in 1993, miRNA has been tightly linked to stage-specific cell fates during embryonic development $[15,16]$. It was really amazing that loss of the single miRNA lin-4, or even its binding site on lin-14, resulted in significant developmental defects in C. elegans $[15,16]$. There is no better support for the importance of miRNAs in development than this evidence. However, the significance of these tiny RNAs was not recognized until 2000, when the second miRNA, let-7, was detected and its sequence and temporal expression was shown to be highly conserved across a wide range of animal species [17,18]. The extensive tiny RNA world was further revealed by the identification of approximately 100 miRNA genes in worms, flies, and human cells in 2001 [19-21].

The global requirement for miRNAs in embryogenesis is evidenced by the genetic disruption of Dicer [22-24], an RNase III-family nuclease that is essential for miRNA biogenesis [25]. While loss of Dicer 1 in mice leads to lethality early in development with the depletion of stem cells [22], zebrafish that lack both maternal and zygotic Dicer activity (known as MZdicer mutants) undergo axis formation and differentiate into multiple cell types but display abnormal morphogenesis during gastrulation, brain formation, somitogenesis, and heart development [24]. Although Dicer has also been implicated in the biogenesis of endogenous small interfering RNAs (siRNAs) [26], many of the defects in MZdicer mutants are rescued by mature miRNA injection, suggesting that the phenotypes are indeed due to an absence of miRNA function [24]. Considering the dual involvement of Dicer, other researchers deleted $\operatorname{Dgcr} 8$, which encodes an RNA-binding protein that is specifically required for miRNA processing [27]. In striking contrast to Dicer, the loss of zygotic Dgcr8 in mice led to embryonic arrest prior to E6.5 but did not impair preimplantation development, indicating that miRNAs primarily play regulatory roles in postimplantation development [28,29].

Although the mechanism for how individual miRNAs exert their specific roles during development is not fully understood, it is clear that miRNAs are primarily associated with the establishment and maintenance of cell identity, as Plasterk and colleagues [30] have provided pictures like artwork that show the temporal and spatial expression patterns of 115 conserved vertebrate miRNAs in zebrafish embryos by microarrays and in situ hybridizations. In the past 10 years, overwhelming experimental studies have evidenced the role of miRNAs in promoting the programs that define the fate and characteristics of developing cells in diverse cellular systems [7,31,32]. In particular, a few of miRNAs highly specifically expressed in certain tissues or cell-lineages are more prominent in regulating cell fate, such as miR-1 [33-35], miR-133 [34-36], miR-223 [37,38], miR-124 [39-42], and miR-122 [43-46]. Almost all of these studies indicate that tissue-specific miRNAs play critical roles in promoting lineage-specific differentiation by directly targeting some key regulatory genes [32]. For example, the muscle-specific miR-1 regulates cardiogenesis by targeting Hand2 [33], whereas miR-1 promotes myogenesis by targeting histone deacetylase 4 (HDAC4) [34]. The myeloid-specific miR-223 regulates progenitor proliferation and granulocyte differentiation by the repression of the transcription factors NFI-A [37] and Mef2c [38]. The neuron-specific miR-124 promotes neuronal differentiation by triggering brain-specific alternative pre-mRNA splicing through targeting polypyrimidine tract binding protein 1 (PTBP1) [40]. Additionally, miR-124 antagonizes the anti-neural REST/SCP1 pathway during the development of the embryonic central nervous system (CNS) [42]. The liver-specific miR-122 inhibits the proliferation and promotes the differentiation of hepatocytes during liver development by targeting cut-like homeobox 1 (CUTL1) [43]. Moreover, several tissue-specific miRNAs, such as miR-1, miR-133, miR-145, miR-143, and miR-9, have been shown to help refine and limit gene expression during lineage commitment of embryonic stem cells (ESCs) [35,47-49], suggesting that miRNAs may have general utility in regulating cell-fate decisions from pluripotent ESCs.

Notably, the requirement of individual miRNAs for differentiation, growth, and organogenesis during development is not equal. For example, Horvitz and colleagues [50] generated mutant $C$. elegans strains that each lack multiple or all members of one of the 15 miRNA families; however, they were surprised to find that mutants for 12 of these families did not display strong synthetic abnormalities, suggesting that some miRNA families have subtle roles during development. The data from miRNA-knockout studies in mice are also consistent with this observation [51]. The impact of individual miRNA knockouts on mouse heart development 
is widely studied, and a dozen miRNA families have been investigated so far [51]. The deletion of $m i R-1-2$ in mice causes $\sim 50 \%$ lethality due to ventricular septal defects (VSDs), in spite of only a $50 \%$ reduction in the overall level of miR-1 expression in these mice [52]. Although mice lacking either $m i R-133 a-1$ or $m i R-133 a-2$ genes do not display obvious cardiac abnormalities, the deletion of both genes results in $\sim 56 \%$ lethality due to VSDs and chamber dilatation [53]. The deletion of the miR-17-92 cluster also causes perinatal lethality due to VSDs [54]. The deletion of endothelial cell-specific miR-126 results in $\sim 40 \%$ lethality due to vascular developmental defects $[55,56]$. In contrast, mice lacking $m i R-208 a[57,58]$ or $m i R-145$ [59] were viable but with different degrees of heart defects. However, no apparent phenotype was observed in mice that lacked $m i R-208 b$ [60], miR-499 [60], miR-143 [59], the miR-106a363 cluster [54], or the $m i R-106 b-25$ cluster [54]. Interestingly, consistent with the data that were obtained from in vitro studies, it has been suggested that tissue-specific and highly abundant miRNAs, such as miR-1, miR-133, and miR-126, are essential for the development of their associated tissues and organs; moderately abundant miRNAs are secondary but required, whereas other miRNAs with lower expression levels may be unnecessary.

\section{Reprogramming the somatic cells into plu- ripotent cells or other lineages by miRNAs}

Whereas "program" in biology stands for a series of developmental and differentiation processes that are orderly arranged in the life cycle of an organism, "reprogram" is the reversal of programming, which refers to the erasure and remodeling of the functional state of the genome, resulting in the conversion of a differentiated cell to an immature cell (pluripotent) or the transit directly from one lineage to another (lineage reprogramming) [61]. Although it has been studied more than 50 years and remarkable breakthroughs were obtained [62-64], only in recent years has cellular reprogramming attracted unprecedented attention, especially after Yamanaka and colleagues $[65,66]$ reported that fibroblasts can be induced into pluripotent stem cells (iPS cells) by the forced expression of four transcription factors (OCT4, KLF4, MYC and SOX2, which are collectively called OKMS). Cellular reprogramming has been achieved by many approaches, such as nuclear transfer, cell fusion, long-term in vitro cell culture, forced expression of transcription factors [67], and, most recently, by the introduction of miRNAs [13]. However, compared with other traditional approaches such as nuclear transfer and cell fusion, the ectopic expression of defined factors has obvious advantages in technical operability and simplicity, which makes it more attractive. From 2006 to the present, accumulating studies have witnessed the success and progress in cellular reprogramming by the forced expression of tran- scription factors $[10,11,68]$ and/or miRNAs [14,69]. Several representative studies in which both transcription factors and miRNAs were employed are schematically shown in Figure 1.

After the recognition of the key roles of miRNAs in cell-fate decision during embryonic development and cellular differentiation, it is not surprising that they are currently emerging as competent players in somatic cell reprogramming [70-72]. In particular, all of the miRNAs that are specifically and/or abundantly expressed in ESCs have also been shown to induce or promote pluripotency $[69,73]$. ESCs express a distinctive set of miRNAs, which are required for the establishment and maintenance of ESC identity $[73,74]$. The majority of these miRNAs are derived from two polycistronic clusters: the miR-302-367 cluster (miR-302a/b/c/d and miR-367) and, in mice, the miR-290295 cluster (miR-290, miR-291a, miR-291b, miR-292, miR-293, miR-294 and miR-295) or, in humans, the miR-371-373 cluster (miR-371, miR-372 and miR-373) [75,76]. Significantly, these ESC-specific miRNAs possess the same or similar 'seed' sequence, and thus, are predicted to cooperatively regulate downstream targets $[77,78]$. These ESC-specific miRNAs account for as much as $60 \%-70 \%$ of the total miRNAs that are expressed in ESCs [77-80]. It has been shown that the miR-302-367 cluster is among the most significantly upregulated miRNAs in both human ESCs and iPS cells compared with fibroblasts [81]. Thus, these ESC-specific miRNAs can strongly promote or directly induce the reprogramming of somatic cells to pluripotency [70-72,82-84]. Mechanistically, these miRNAs have been shown to regulate various cellular processes, including cell cycle, differentiation, mesenchymal-to-epithelial transition (MET), epigenetic regulation, cell signaling and vesicular transport [82,85-91], all of which are critical for the establishment and maintenance of pluripotency.

Another notably important miRNA family that promotes iPS is the miR-17 family, which is not ESCs-specific but is enriched in pluripotent cells $[77,78]$. One of the main defects in miRNA-null ESCs is a reduced proliferation rate, as shown in $\mathrm{Dgcr}{ }^{-/-}$ESCs [28]. A functional screen identified several miRNAs that were able to rescue this defect, which are primarily transcribed from the ESC-specific miR-290295 and miR-302-367 clusters, as well as four miRNAs from the miR-17 family [85]. The miR-17 family consists of three paralogous polycistronic clusters on different chromosomes: miR-17-92 (miR-17, miR-18a, miR-19a, miR-20a, miR-19b-1, and miR-92a-1), miR-106b-25 (miR-106b, miR-93, and miR-25), and miR-106a-363 (miR-106a, miR-18b, miR-20b, miR-19b-2, miR-92a-2, and miR-363) [92]. Several members of this family were shown to be highly induced during early reprogramming and greatly enhanced the induction of iPS cells [93], most likely by regulating cell cycle progression $[85,94,95]$ and the MET step in the initiation stage of reprogramming [93].

Apart from these so-called ESCC miRNAs (ESC-specific 


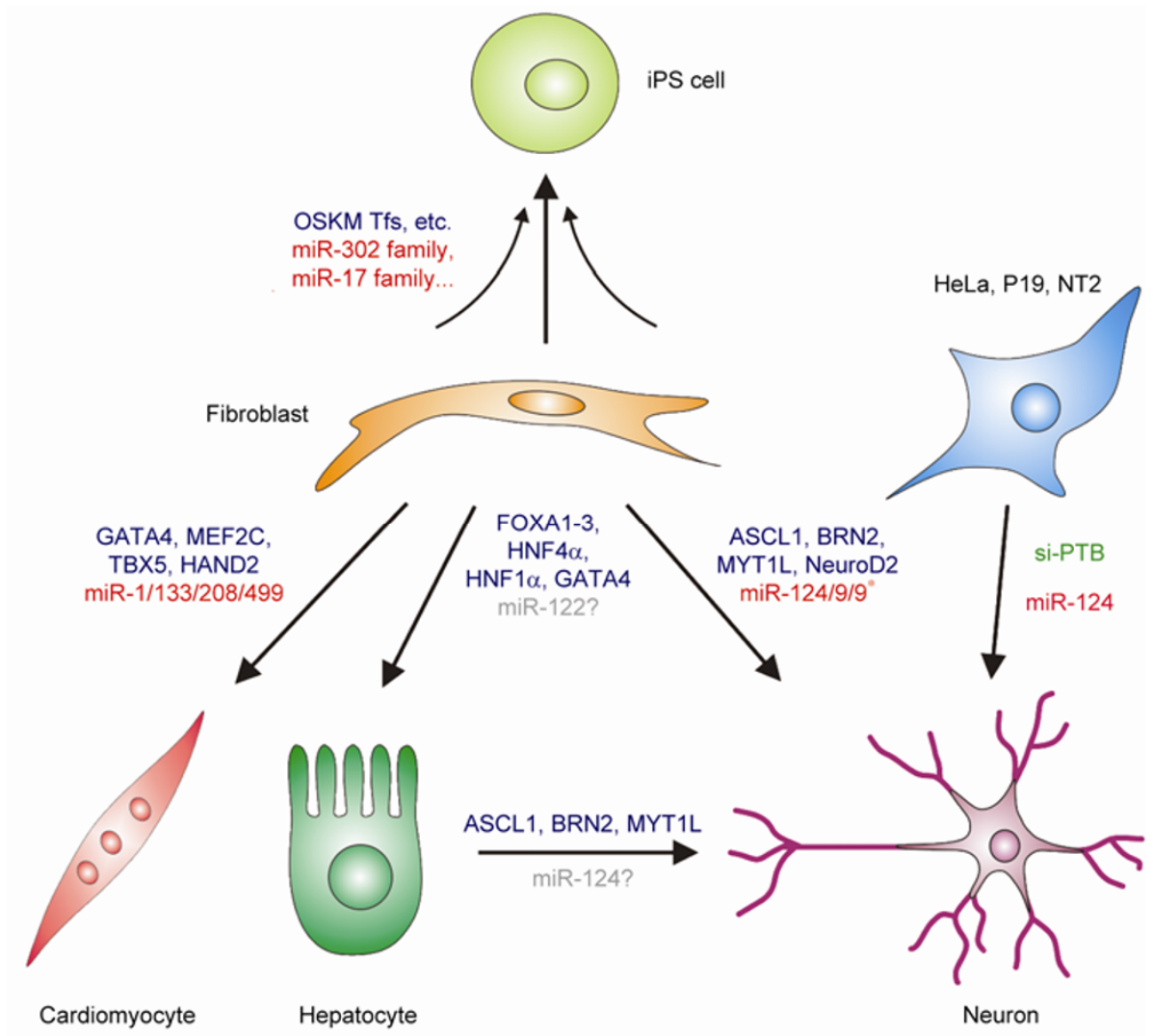

Figure 1 miRNAs that are involved in cell reprogramming. The schematic illustrates the most studied cell types in somatic cell reprogramming, including iPS cells, neurons, cardiomyocytes and hepatocytes. The first three cell types have been successfully reprogrammed from fibroblasts, either by transcription factors or miRNAs that are specifically expressed in certain cell types. Reprogrammed hepatocytes have also been successfully generated by the enforced expression of transcription factors. Although unverified, miR-122 is expected to have the potential to convert fibroblasts into hepatocytes and is therefore shown in gray letters. Similarly, miR-124 is expected to be capable of reprogramming hepatocytes into neurons and is also shown in gray.

cell cycle-regulating miRNAs) [85], many other miRNA families also facilitate iPS [69,96]. The miR-200 family (miR-200a, miR-200b, miR-200c, miR-141 and miR-429) and miR-205 are shown to be induced during the initiation phase of reprogramming, which stimulate MET and promote mouse embryonic fibroblast (MEF) reprogramming, probably by targeting Zeb1, Zeb2, Snail and Slug [97,98]. Significantly, mir-200c, combined with mir-302 and mir-369 family miRNAs, has been shown to successfully induce the reprogramming of mouse and human cells to pluripotency [72]. A functional miRNA screen identified the miR-130 family (miR-130/301/721), which enhanced iPS generation through targeting the homeobox transcription factor Meox2 (also known as Gax) [99]. miR-138 is shown to promote iPS generation through the regulation of the p53 signaling pathway [100]. Recently, miR-29b has been shown to be a mediator of Sox 2 function and is required for OSKM-mediated reprogramming through targeting Dnmt3a and Dnmt3b [101]. However, miR-29a was previously shown to negatively regulate iPS [102]. This difference might be cell context-dependent and should be further investigated.

In addition to the miRNA families that are enriched in pluripotent cells, there are also several miRNA families that are expressed at significantly lower levels in pluripotent cells than in differentiated cells and thus play a negative role in iPS generation [69]. The most remarkable one is the let-7 family, a group of well-known differentiation-promoting miRNAs that are highly enriched in differentiated cells compared with ESCs and iPS cells [77,81]. Coincidentally, in contrast to ESC-specific miRNAs, the let-7 family targets Lin-28, c-Myc, N-Myc and many other pluripotent genes to inhibit ESC self-renewal and promote differentiation [88]. Additionally, a group of miRNAs, which include miR-134, miR-296, miR-470 [103], miR-145 [48] and the miR-34 family [104], directly target different pluripotent transcription factors (OCT4, SOX2, KLF4, Nanog, N-Myc, etc.) and therefore provide a barrier for the reprogramming to pluripotency. In addition, miR-199 [105], miR-21 and miR-29a [102] have also been shown to inhibit reprogramming. Moreover, a few miRNAs, such as the miR-23 family (miR-23a, miR-23b, miR-27a, miR-27b) and miR-143, are significantly downregulated in pluripotent cells and are therefore expected to inhibit somatic cell reprogramming [81].

Direct lineage reprogramming (also known as transdif- 
ferentiation) was first observed 25 years ago [63], and has become one of the most attractive fields in cell biology and regenerative medicine [106]. Recent studies have demonstrated that a diverse range of cell types, such as pancreatic $\beta$ cells [107], neurons [108,109], cardiomyocytes [110-112], and hepatocytes $[113,114]$, can be directly induced from somatic cells using cocktails of transcription factors. Remarkably, an important breakthrough was made in 2010, when, for the first time, transdifferentiation across germ layers-conversion of mouse fibroblasts (mesoderm) to neurons (ectoderm) was achieved [108]. Similar to that in the reprogramming of pluripotent cells, transcription factors are widely employed in the induction of transdifferentiation and the number of successful studies is constantly increasing $[61,115]$. Interestingly, two groups of tissue-specific miRNAs have been shown to accomplish this mission as excellently as transcription factors [116,117]. Crabtree and colleagues [116] showed that miR-124 and miR-9/9* alone, which are selectively expressed in post-mitotic neurons, could induce the conversion of fibroblasts to neurons. This exciting success is based on their previous finding that miR-124 and miR-9* play a critical role in neural development by the repression of BAF53a and thereby mediate a switch of chromatin-remodeling complexes [118]. In addition, miR124 cooperates with two transcription factors (MYT1L and BRN2), which are able to reprogram fibroblasts to functional neurons [119]. In the latter instance, a combination of four cardiomyocytes-specific miRNAs, miR-1, miR-133, miR-208, and miR-499, is capable of inducing the direct cellular reprogramming of fibroblasts to cardiomyocyte-like cells [117]. Recently, it has been shown that four human cardiac transcription factors, including GATA4, Hand2, T-box5, and myocardin, and two miRNAs, miR-1 and miR-133, are necessary and sufficient for the direct myocardial reprogramming of human fibroblasts [120]. Although successful instances of transdifferentiation mediated by miRNAs are currently few, some other tissue-specific miRNAs are also expected to be capable of promoting transdifferentiation to their respective cell types. Among the most promising candidates is hepatocyte-specific miR-122, which has been shown to activate the expression of several hepatocyte functional genes in a hepatoblastoma cell line [43].

Recently, a remarkable study demonstrated that the repression of a single RNA-binding polypyrimidine tractbinding (PTB) protein is sufficient to induce the transdifferentiation of multiple cell types of diverse origin into neuronal-like cells or even functional neurons [121]. This unsuspected phenomenon appears to be due to the relief of a PTB-mediated blockade the action of miRNA (miR-124) on several components of the REST (RE1-silencing transcription factor) complex and the subsequent derepression of neuronal-specific genes and genes that encode miRNAs [121]. Interestingly, the authors further provide evidence that the knockdown of SCP1 or REST is sufficient to trigger the transdifferentiation of MEFs into neuronal-like cells [121]. Therefore, this finding suggests that a conserved regulatory network, which is constituted by lineage-specific miRNAs, transcription factors, and epigenetic regulators, as well as other unrecognized factors, functions across diverse cell types; therefore, the artificial alteration of one of these factors might mediate cell reprogramming.

\section{Modality by which miRNAs specify a cell fate}

How is a specific cell state regulated by hundreds of miRNAs? Using ESCs as a model, we will demonstrate this mechanism because the miRNA expression profiles, functions, and targets, all have been well-studied in ESCs. Based on the aforementioned evidence, we provide a "Helm" model of the role of miRNAs in the establishment and maintenance of cellular homeostasis (Figure 2). According to this model, the cell identity (function and fate) of a given cell is controlled by a series of balances between miRNAs and their targets. The status of each miRNA family is associated with their relative abundance in the cells, as illustrated in Figure 2.

Several characteristics should be noted in this model. (i) The unity of "yin and yang". All the miRNA families can be divided into three groups on the basis of their attitude to the present state: positive, negative and neutral. miRNAs that are essential for the establishment and maintenance of current state are positive members ("yang"), whereas miRNAs that inhibit the current state are negative members ("yin"), leaving miRNAs that do not influence the current state as the neutral members. In ESCs, the miR-302 family (the miR-302-367 cluster, the miR-371-373 cluster, and the miR-290-295 cluster in mice), the miR-17 family (the miR-17-92 cluster, the miR-106b-25 cluster and the miR-106a-363 cluster), the miR-200 family, the miR-130 family, miR-138, etc., which promote pluripotency, are "yang". In contrast, tissue-specific miRNAs, such as miR-124/9, miR-1/133, and miR-122, the let-7 family, the miR-34 family, miR-145, miR-134, miR-296, miR-470, miR-199, etc., which promote differentiation and inhibit pluripotency, are "yin". Correspondingly, the "yin and yang" of target genes is opposite to miRNAs. For example, the pluripotent transcription factors OCT4, SOX2, KLF4, Nanog and N-Myc are targets of miR-145 or the miR-34 family, and accordingly are "yang". (ii) The dose-dependent effect. Each balance has a unique tendency, which is dependent on the relative level of both miRNAs and their targets. When miRNAs are highly abundant, such as the miR-302 family, their targets are fully repressed and the balance tends to miRNAs. In addition, the miR-17-92 cluster is relatively enriched, and therefore, their targets are significantly repressed. In contrast, when miRNAs are expressed at low levels or silenced, such as tissue-specific miRNAs and the let-7 family, their targets are fully expressed and the balance tends to target proteins. Moreover, 


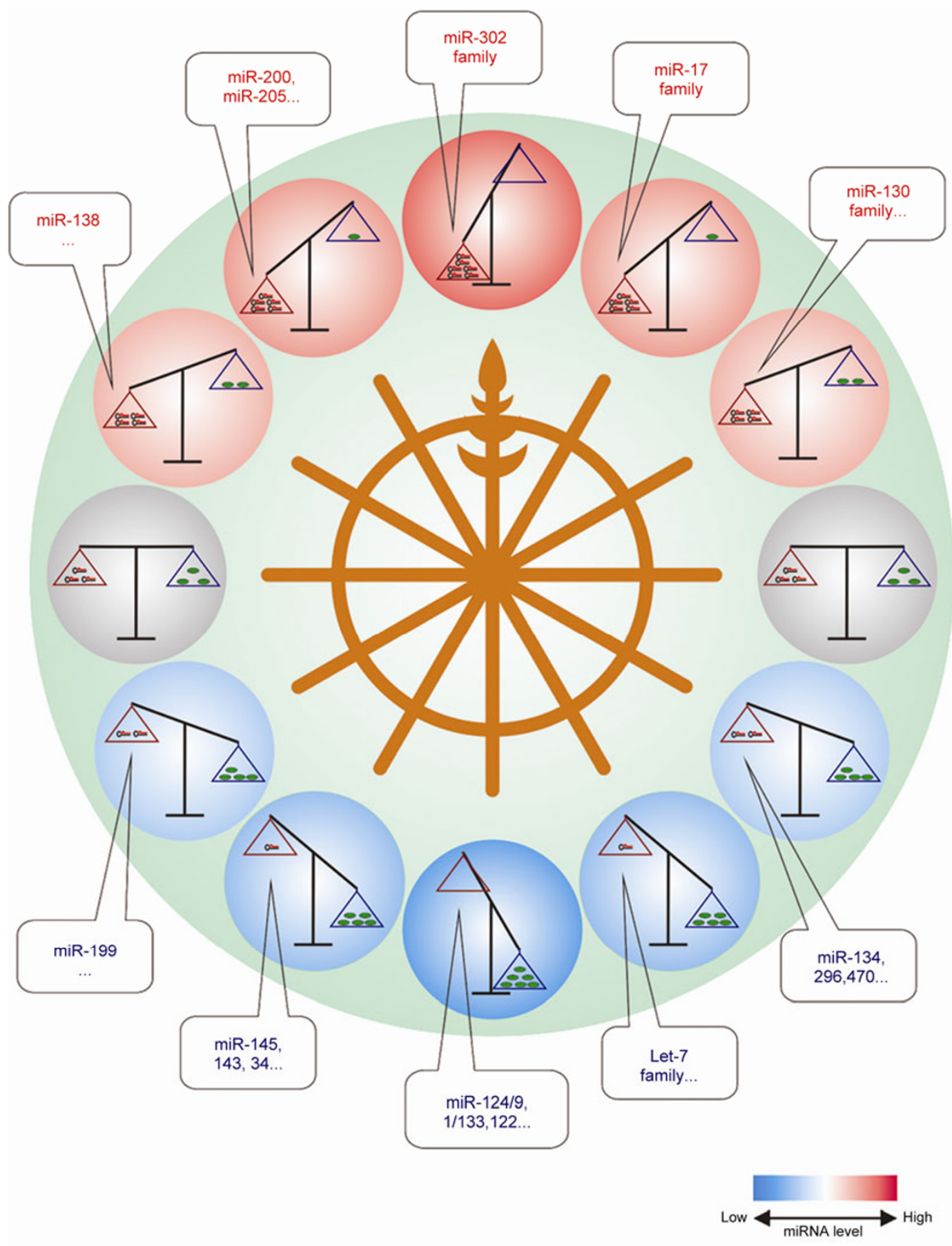

Figure 2 The Helm model showing the weight of different miRNA families in controlling the cell fate, using miRNAs that are involved in regulating the pluripotency and differentiation of ESCs as examples. ESCs express hundreds of miRNAs that work synergistically to direct the "course" of ESCs and to keep ESCs on the route of pluripotency, by a series of balances between miRNAs and their targets. However, the contribution of each miRNA family to pluripotency is different. Because of its overwhelming abundance, the miR-302 family takes the helm of ESCs. The next enriched family is the miR-17 family, which serves as "the mate" and escort for pluripotency. Other miRNA families, such as the miR-200 family, the miR-130 family and miR-138, also facilitate pluripotency by regulating different targets. In contrast, some miRNA families that are highly expressed in somatic cells but are expressed at low levels or silenced in ESCs can be regarded as "opposition", which directly or indirectly inhibit pluripotency. Tissue-specific miRNAs, such as miR-124/9, miR-1/133, and miR-122, are the predominant miRNA families in specific somatic cells (neurons, muscles, and hepatocytes, respectively) and consequently lead the "opposition". The let-7 family miRNAs are highly enriched in most somatic cells and thus are important forces in the "opposition". The miR-34 family, miR-143, miR-145, miR-134, miR-296, miR-470, miR-199, etc. also set obstacles for pluripotency. The hairpins in the red triangles represent miRNAs, and the green ovals in blue triangles represent target proteins. Gradient colors of balls indicate the relative expression levels for each miRNA family: blue, low; gray, mean; red, high. 
there are some balances in which the expression of both miRNAs and their targets are moderate, and thus, the balance is level. (iii) Different weights. The importance and contribution of each miRNA family are not equal. Highly enriched miRNAs usually play a dominant role in the establishment and maintenance of the current cell state, whereas the contribution of miRNAs that are expressed at significantly lower levels might be negligible. Many examples have been discussed in the text above. (iv) Independence and interaction. The regulation of each balance may be independent. However, changes in some critical balances may have a significant impact on the other balances through cross-talk. For example, the role of the miR-302 family is oppositely related to the let-7 family, and therefore, they could indirectly repress the expression of each other. (v) Homeostasis is changeable. When the dominant miRNA families are substituted by specific miRNAs in other cell types, as observed in miRNA-mediated lineage transdifferentiation and iPS, the cell fate could be reprogrammed and a new homeostasis will form.

All of this evidence points to the distinctive importance of miRNAs that are specifically and abundantly expressed in specific cells. There are several possible reasons that may explain why the functional role in development and cell reprogramming has been primarily ascribed to the most enriched miRNAs. First, there is an obvious dosage requirement for miRNAs to efficiently repress the expression of target genes, as evidenced by the phenomenon that almost all target genes are gradually downregulated with the increased level of related miRNAs $[33,43]$. Second, an abundance of copies of mature miRNAs also seems to be required for the direct repression of a large number of genes [122]. Third, tissue-specific miRNAs are expected to regulate switch genes specifying the cell fate and developmental timing because many transcription factors, particularly transcription repressors, have been shown to be targeted by tissue-specific miRNAs $[33,34,37,42,43]$. Last, tissuespecific miRNAs are generally woven into the principal regulatory network, which is constituted by enriched transcription factors and the dominant signaling pathway $[43,123,124]$.

\section{Conclusion and perspectives}

Whereas a programmed life cycle is a great masterpiece of natural evolution over billions of years, cell reprogramming, with its promising application in regenerative medicine, is a creative result of human civilization. Although small, miRNAs have a high potential for use in cell reprogramming for the following reasons. First, miRNAs can be synthesized by high-throughput and easily introduced into a variety of cell types by transfection, significantly reducing the technical requirements. Second, using mature miRNAs only transiently can eliminate the risks and concerns of pos- sible genomic disruption by viral integration. Last, most lineage-specific miRNAs in adult tissues have been shown to play a tumor-suppressing role; thus, the oncogenesis potentiated by gene overexpression can be reduced.

Understanding the molecular mechanisms that underlie programming and reprogramming processes is crucial for the creation of high-quality reprogrammed cells and may be useful for therapeutic applications [125]. Despite the great advances obtained, we are only about to understand the molecular basis of these processes, primarily because it is currently challenging to decipher the functional targets of miRNAs which usually regulate a large number of genes to modulate biological networks. Fortunately, newly developed high-throughput RNA capturing and sequencing technologies may assist in determining the key targets of miRNAs from hundreds of candidates [126-128]. For example, using Argonaute CLIP-Seq (sequencing of RNAs isolated by crosslinking immunoprecipitation), Darnell and colleagues [126] provided the first in vivo miRNA-mRNA interaction map in mouse brain and identified a large target-network that may account for the regulatory role of miR-124. Two independent groups studied the genomewide miRNA-target interactions in mouse and human ESCs, respectively, and identified a large group of targets of ESC-specific miRNA families [129,130]. Functional studies identified novel roles of the miR-302 family in maintaining pluripotency and in regulating hESC differentiation, partly through promoting the bone morphogenetic protein (BMP) signaling [130], which is also necessary for the MET during the initiation phase of somatic cell reprogramming [97].

Waddington's concept of the 'epigenetic landscape' has served as an educative hierarchical model to illustrate the progressive restriction of cell differentiation potential during normal development [131]. However, this concept fails to explain the success in somatic cell reprogramming. Most recently, Brüstle and colleagues have proposed a nonhierarchical 'epigenetic disc' model in which the pluripotent state is just one of many possible states, which is not any higher in hierarchy than other cell states and, therefore, can be bypassed in the process of cell fate conversion [14]. Consistent with this viewpoint, our "Helm" model also implies that cell fate is controlled in the hands of a few highly enriched miRNAs; therefore, cell fate can theoretically be converted by the substitution of the predominating miRNA families with lineage-specific miRNAs in target cells. However, this prospect should be further explored by extensive investigations.

This work was supported by the National Natural Science Foundation of China (31200593, 31230042, 81070589), the Guangdong Natural Science Foundation (S2011040001760), the Fundamental Research Funds for the Central Universities (13lgpy40), and the National Basic Research Program of China (2011CB811300).

1 He L, Hannon G J. microRNAs: Small RNAs with a big role in gene 
regulation. Nat Rev Genet, 2004, 5: 522-531

2 Carthew R W, Sontheimer E J. Origins and mechanisms of miRNAs and siRNAs. Cell, 2009, 136: 642-655

3 Ghildiyal M, Zamore P D. Small silencing RNAs: An expanding universe. Nat Rev Genet, 2009, 10: 94-108

4 Ulitsky I, Bartel D P. lincRNAs: Genomics, evolution, and mechanisms. Cell, 2013, 154: 26-46

5 Salmena L, Poliseno L, Tay Y, et al. A ceRNA hypothesis: The Rosetta Stone of a hidden RNA language? Cell, 2011, 146: 353358

6 Pasquinelli A E. microRNAs and their targets: Recognition, regulation and an emerging reciprocal relationship. Nat Rev Genet, 2012, 13: 271-282

7 Pauli A, Rinn J L, Schier A F. Non-coding RNAs as regulators of embryogenesis. Nat Rev Genet, 2011, 12: 136-149

8 Esteller M. Non-coding RNAs in human disease. Nat Rev Genet, 2011, 12: 861-874

9 Ambros V. microRNAs and developmental timing. Curr Opin Genet Dev, 2011, 21: 511-517

10 Stadtfeld M, Hochedlinger K. Induced pluripotency: History, mechanisms, and applications. Gene Dev, 2010, 24: 2239-2263

11 Vierbuchen T, Wernig M. Molecular roadblocks for cellular reprogramming. Mol Cell, 2012, 47: 827-838

12 Shenoy A, Blelloch R. microRNA induced transdifferentiation. F1000 Biol Rep, 2012, 4: 3

13 Bao X C, Zhu X H, Liao B J, et al. microRNAs in somatic cell reprogramming. Curr Opin Cell Biol, 2013, 25: 208-214

14 Ladewig J, Koch P, Brüstle O. Leveling Waddington: The emergence of direct programming and the loss of cell fate hierarchies. Nat Rev Mol Cell Bio, 2013, 14: 225-236

15 Lee R C, Feinbaum R L, Ambros V. The C. elegans heterochronic gene lin-4 encodes small RNAs with antisense complementarity to lin-14. Cell, 1993, 75: 843-854

16 Wightman B, Ha I, Ruvkun G. Posttranscriptional regulation of the heterochronic gene lin-14 by lin- 4 mediates temporal pattern formation in C. elegans. Cell, 1993, 75: 855-862

17 Reinhart B J, Slack F J, Basson M, et al. The 21-nucleotide let-7 RNA regulates developmental timing in Caenorhabditis elegans. Nature, 2000, 403: 901-906

18 Pasquinelli A E, Reinhart B J, Slack F, et al. Conservation of the sequence and temporal expression of let-7 heterochronic regulatory RNA. Nature, 2000, 408: 86-89

19 Lagos-Quintana M, Rauhut R, Lendeckel W, et al. Identification of novel genes coding for small expressed RNAs. Science, 2001, 294: 853-858

20 Lau N C, Lim L P, Weinstein E G, et al. An abundant class of tiny RNAs with probable regulatory roles in Caenorhabditis elegans. Science, 2001, 294: 858-862

21 Lee R C, Ambros V. An extensive class of small RNAs in Caenorhabditis elegans. Science, 2001, 294: 862-864

22 Bernstein E, Kim S Y, Carmell M A, et al. Dicer is essential for mouse development. Nat Genet, 2003, 35: 215-217

23 Wienholds E, Koudijs M J, van Eeden F J M, et al. The microRNAproducing enzyme Dicer1 is essential for zebrafish development. Nat Genet, 2003, 35: 217-218

24 Giraldez A J, Cinalli R M, Glasner M E, et al. microRNAs regulate brain morphogenesis in zebrafish. Science, 2005, 308: 833-838

25 Hutvagner G, McLachlan J, Pasquinelli A E, et al. A cellular function for the RNA-interference enzyme Dicer in the maturation of the let-7 small temporal RNA. Science, 2001, 293: 834-838

26 Babiarz J E, Ruby J G, Wang Y M, et al. Mouse ES cells express endogenous shRNAs, siRNAs, and other Microprocessorindependent, Dicer-dependent small RNAs. Gene Dev, 2008, 22: 2773-2785

27 Gregory R I, Yan K P, Amuthan G, et al. The microprocessor complex mediates the genesis of microRNAs. Nature, 2004, 432: 235-240

28 Wang Y M, Medvid R, Melton C, et al. DGCR8 is essential for microRNA biogenesis and silencing of embryonic stem cell self- renewal. Nat Genet, 2007, 39: 380-385

29 Suh N, Baehner L, Moltzahn F, et al. microRNA function is globally suppressed in mouse oocytes and early embryos. Curr Biol, 2010, 20: 271-277

30 Wienholds E, Kloosterman W P, Miska E, et al. microRNA expression in zebrafish embryonic development. Science, 2005, 309: 310-311

31 Stefani G, Slack F J. Small non-coding RNAs in animal development. Nat Rev Mol Cell Bio, 2008, 9: 219-230

32 Ivey K N, Srivastava D. microRNAs as regulators of differentiation and cell fate decisions. Cell Stem Cell, 2010, 7: 36-41

33 Zhao Y, Samal E, Srivastava D. Serum response factor regulates a muscle-specific microRNA that targets Hand 2 during cardiogenesis. Nature, 2005, 436: 214-220

34 Chen J F, Mandel E M, Thomson J M, et al. The role of microRNA-1 and microRNA-133 in skeletal muscle proliferation and differentiation. Nat Genet, 2006, 38: 228-233

35 Ivey $\mathrm{K} \mathrm{N}$, Muth A, Amold J, et al. microRNA regulation of cell lineages in mouse and human embryonic stem cells. Cell Stem Cell, 2008, 2: 219-229

36 Care A, Catalucci D, Felicetti F, et al. microRNA-133 controls cardiac hypertrophy. Nat Med, 2007, 13: 613-618

37 Fazi F, Rosa A, Fatica A, et al. A minicircuitry comprised of microRNA-223 and transcription factors NFI-A and C/EBP alpha regulates human granulopoiesis. Cell, 2005, 123: 819-831

38 Johnnidis J B, Harris M H, Wheeler R T, et al. Regulation of progenitor cell proliferation and granulocyte function by microRNA223. Nature, 2008, 451: 1125-1129

39 Krichevsky A M, Sonntag K C, Isacson O, et al. Specific microRNAs modulate embryonic stem cell-derived neurogenesis. Stem Cells, 2006, 24: 857-864

40 Makeyev E V, Zhang J W, Carrasco M A, et al. The microRNA miR-124 promotes neuronal differentiation by triggering brainspecific alternative Pre-mRNA splicing. Mol Cell, 2007, 27: 435448

41 Cao X W, Pfaff S L, Gage F H. A functional study of miR-124 in the developing neural tube. Gene Dev, 2007, 21: 531-536

42 Visvanathan $\mathrm{J}$, Lee $\mathrm{S}$, Lee $\mathrm{B}$, et al. The microRNA miR-124 antagonizes the anti-neural REST/SCP1 pathway during embryonic CNS development. Gene Dev, 2007, 21: 744-749

$43 \mathrm{Xu} \mathrm{H}$, He J H, Xiao Z D, et al. Liver-enriched transcription factors regulate microRNA-122 that targets CUTL1 during liver development. Hepatology, 2010, 52: 1431-1442

44 Laudadio I, Manfroid I, Achouri Y, et al. A feedback loop between the liver-enriched transcription factor network and miR-122 controls hepatocyte differentiation. Gastroenterology, 2012, 142: 119-129

45 Hsu S H, Wang B, Kota J, et al. Essential metabolic, antiinflammatory, and anti-tumorigenic functions of miR-122 in liver. $\mathrm{J}$ Clin Invest, 2012, 122: 2871-2883

46 Tsai W C, Hsu S D, Hsu C S, et al. microRNA-122 plays a critical role in liver homeostasis and hepatocarcinogenesis. J Clin Invest, 2012, 122: 2884-2897

47 Cordes K R, Sheehy N T, White M P, et al. miR-145 and miR-143 regulate smooth muscle cell fate and plasticity. Nature, 2009, 460: $705-710$

48 Xu N, Papagiannakopoulos T, Pan G, et al. microRNA-145 regulates OCT4, SOX2, and KLF4 and represses pluripotency in human embryonic stem cells. Cell, 2009, 137: 647-658

49 Delaloy C, Liu L, Lee J A, et al. microRNA-9 coordinates proliferation and migration of human embryonic stem cell-derived neural progenitors. Cell Stem Cell, 2010, 6: 323-335

50 Alvarez-Saavedra E, Horvitz H R. Many families of $C$. elegans microRNAs are not essential for development or viability. Curr Biol, 2010, 20: 367-373

51 Liu N, Olson E N. microRNA regulatory networks in cardiovascular development. Dev Cell, 2010, 18: 510-525

52 Zhao Y, Ransom J F, Li A, et al. Dysregulation of cardiogenesis, cardiac conduction, and cell cycle in mice lacking miRNA-1-2. Cell, 2007, 129: 303-317 
53 Liu N, Bezprozvannaya S, Williams A H, et al. microRNA-133a regulates cardiomyocyte proliferation and suppresses smooth muscle gene expression in the heart. Gene Dev, 2008, 22: 3242-3254

54 Ventura A, Young A G, Winslow M M, et al. Targeted deletion reveals essential and overlapping functions of the miR-17 similar to 92 family of miRNA clusters. Cell, 2008, 132: 875-886

55 Wang S S, Aurora A B, Johnson B A, et al. The endothelial-specific microRNA miR-126 governs vascular integrity and angiogenesis. Dev Cell, 2008, 15: 261-271

56 Kuhnert F, Mancuso M R, Hampton J, et al. Attribution of vascular phenotypes of the murine Egfl7 locus to the microRNA miR-126. Development, 2008, 135: 3989-3993

57 van Rooij E, Sutherland L B, Qi X X, et al. Control of stressdependent cardiac growth and gene expression by a microRNA. Science, 2007, 316: 575-579

58 Callis T E, Pandya K, Seok H Y, et al. microRNA-208a is a regulator of cardiac hypertrophy and conduction in mice. $\mathrm{J}$ Clin Invest, 2009, 119: 2772-2786

59 Xin M, Small E M, Sutherland L B, et al. microRNAs miR-143 and miR-145 modulate cytoskeletal dynamics and responsiveness of smooth muscle cells to injury. Gene Dev, 2009, 23: 2166-2178

60 van Rooij E, Quiat D, Johnson B A, et al. A family of microRNAs encoded by myosin genes governs myosin expression and muscle performance. Dev Cell, 2009, 17: 662-673

61 Zhou J N, Yue W, Pei X T. Advances in cell lineage reprogramming. Sci China Life Sci, 2013, 56: 228-233

62 Gurdon J B. The developmental capacity of nuclei taken from intestinal epithelium cells of feeding tadpoles. J Embryol Exp Morphol, 1962, 10: 622-640

63 Davis R L, Weintraub H, Lassar A B. Expression of a single transfected cDNA converts fibroblasts to myoblasts. Cell, 1987, 51: 987-1000

64 Wilmut I, Schnieke A E, McWhir J, et al. Viable offspring derived from fetal and adult mammalian cells. Nature, 1997, 385: 810-813

65 Takahashi K, Yamanaka S. Induction of pluripotent stem cells from mouse embryonic and adult fibroblast cultures by defined factors. Cell, 2006, 126: 663-676

66 Takahashi K, Tanabe K, Ohnuki M, et al. Induction of pluripotent stem cells from adult human fibroblasts by defined factors. Cell, 2007, 131: 861-872

67 Yamanaka S. Strategies and new developments in the generation of patient-specific pluripotent stem cells. Cell Stem Cell, 2007, 1: 39_ 49

68 Chen L Y, Liu L. Current progress and prospects of induced pluripotent stem cells. Sci China Ser C-Life Sci, 2009, 52: 622-636

69 Greve T S, Judson R L, Blelloch R. microRNA control of mouse and human pluripotent stem cell behavior. Annu Rev Cell Dev Biol, 2013, doi: 10.1146/annurev-cellbio-101512-122343

70 Judson R L, Babiarz J E, Venere M, et al. Embryonic stem cellspecific microRNAs promote induced pluripotency. Nat Biotechnol, 2009, 27: 459-461

71 Anokye-Danso F, Trivedi C M, Juhr D, et al. Highly efficient miRNA-mediated reprogramming of mouse and human somatic cells to pluripotency. Cell Stem Cell, 2011, 8: 376-388

72 Miyoshi N, Ishii H, Nagano H, et al. Reprogramming of mouse and human cells to pluripotency using mature microRNAs. Cell Stem Cell, 2011, 8: 633-638

73 Leonardo T R, Schultheisz H L, Loring J F, et al. The functions of microRNAs in pluripotency and reprogramming. Nat Cell Biol, 2012, 14: 1114-1121

74 Martinez N J, Gregory R I. microRNA gene regulatory pathways in the establishment and maintenance of ESC identity. Cell Stem Cell, 2010, 7: 31-35

75 Houbaviy H B, Murray M F, Sharp P A. Embryonic stem cellspecific microRNAs. Dev Cell, 2003, 5: 351-358

76 Suh M R, Lee Y, Kim J Y, et al. Human embryonic stem cells express a unique set of microRNAs. Dev Biol, 2004, 270: 488-498

77 Marson A, Levine S S, Cole M F, et al. Connecting microRNA genes to the core transcriptional regulatory circuitry of embryonic stem cells. Cell, 2008, 134: 521-533

78 Laurent L C, Chen J, Ulitsky I, et al. Comprehensive microRNA profiling reveals a unique human embryonic stem cell signature dominated by a single seed sequence. Stem Cells, 2008, 26: 15061516

79 Bar M, Wyman S K, Fritz B R, et al. microRNA discovery and profiling in human embryonic stem cells by deep sequencing of small RNA libraries. Stem Cells, 2008, 26: 2496-2505

80 Morin R D, O'Connor M D, Griffith M, et al. Application of massively parallel sequencing to microRNA profiling and discovery in human embryonic stem cells. Genome Res, 2008, 18: 610-621

81 Wilson K D, Venkatasubrahmanyam S, Jia F J, et al. microRNA profiling of human-induced pluripotent stem Cells. Stem Cells Dev, 2009, 18: 749-757

82 Subramanyam D, Lamouille S, Judson R L, et al. Multiple targets of miR-302 and miR-372 promote reprogramming of human fibroblasts to induced pluripotent stem cells. Nat Biotechnol, 2011, 29: 443448

83 Lin S L, Chang D C, Chang-Lin S, et al. Mir-302 reprograms human skin cancer cells into a pluripotent ES-cell-like state. RNA, 2008, 14: 2115-2124

84 Lin S L, Chang D C, Lin C H, et al. Regulation of somatic cell reprogramming through inducible mir-302 expression. Nucleic Acids Res, 2011, 39: 1054-1065

85 Wang Y, Baskerville S, Shenoy A, et al. Embryonic stem cellspecific microRNAs regulate the G1-S transition and promote rapid proliferation. Nat Genet, 2008, 40: 1478-1483

86 Sinkkonen L, Hugenschmidt T, Berninger P, et al. microRNAs control de novo DNA methylation through regulation of transcriptional repressors in mouse embryonic stem cells. Nat Struct Mol Biol, 2008, 15: 259-267

87 Benetti R, Gonzalo S, Jaco I, et al. A mammalian microRNA cluster controls DNA methylation and telomere recombination via Rbl2-dependent regulation of DNA methyltransferases. Nat Struct Mol Biol, 2008, 15: 268-279

88 Melton C, Judson R L, Blelloch R. Opposing microRNA families regulate self-renewal in mouse embryonic stem cells. Nature, 2010, 463: 621-626

89 Liao B, Bao X, Liu L, et al. microRNA cluster 302-367 enhances somatic cell reprogramming by accelerating a mesenchymal-toepithelial transition. J Biol Chem, 2011, 286: 17359-17364

90 Luningschror P, Stocker B, Kaltschmidt B, et al. miR-290 cluster modulates pluripotency by repressing canonical NF-kappaB signaling. Stem Cells, 2012, 30: 655-664

91 Wang Y, Melton C, Li Y P, et al. miR-294/miR-302 promotes proliferation, suppresses G1-S restriction point, and inhibits ESC differentiation through separable mechanisms. Cell Rep, 2013, 4: 99-109

92 Tanzer A, Stadler P F. Molecular evolution of a microRNA cluster. J Mol Biol, 2004, 339: 327-335

93 Li Z H, Yang C S, Nakashima K, et al. Small RNA-mediated regulation of iPS cell generation. EMBO J, 2011, 30: 823-834

94 Ivanovska I, Ball A S, Diaz R L, et al. microRNAs in the miR-106b family regulate $\mathrm{p} 21 / \mathrm{CDKN} 1 \mathrm{~A}$ and promote cell cycle progression. Mol Cell Biol, 2008, 28: 2167-2174

95 Petrocca F, Visone R, Onelli M R, et al. E2F1-regulated microRNAs impair TGF beta-dependent cell-cycle arrest and apoptosis in gastric cancer. Cancer Cell, 2008, 13: 272-286

96 Guan D, Zhang W, Zhang W, et al. Switching cell fate, ncRNAs coming to play. Cell Death Dis, 2013, 4: e464

97 Samavarchi-Tehrani P, Golipour A, David L, et al. Functional genomics reveals a BMP-driven mesenchymal-to-epithelial transition in the initiation of somatic cell reprogramming. Cell Stem Cell, 2010, 7: 64-77

98 Wang G Y, Guo X D, Hong W J, et al. Critical regulation of miR-200/ZEB2 pathway in Oct4/Sox2-induced mesenchymal-toepithelial transition and induced pluripotent stem cell generation. Proc Natl Acad Sci USA, 2013, 110: 2858-2863

99 Pfaff N, Fiedler J, Holzmann A, et al. miRNA screening reveals a 
new miRNA family stimulating iPS cell generation via regulation of Meox2. EMBO Rep, 2011, 12: 1153-1159

100 Ye D, Wang G Y, Liu Y, et al. miR-138 promotes induced pluripotent stem cell generation through the regulation of the P53 signaling. Stem Cells, 2012, 30: 1645-1654

101 Guo X D, Liu Q D, Wang G Y, et al. microRNA-29b is a novel mediator of Sox2 function in the regulation of somatic cell reprogramming. Cell Res, 2013, 23: 142-156

102 Yang C S, Li Z H, Rana T M. microRNAs modulate iPS cell generation. RNA, 2011, 17: 1451-1460

103 Tay Y, Zhang J, Thomson A M, et al. microRNAs to Nanog, Oct4 and Sox 2 coding regions modulate embryonic stem cell differentiation. Nature, 2008, 455: 1124-1128

104 Choi Y J, Lin C P, Ho J J, et al. miR-34 miRNAs provide a barrier for somatic cell reprogramming. Nat Cell Biol, 2011, 13: 13531360

105 Wang J, He Q, Han C, et al. p53-facilitated miR-199a-3p regulates somatic cell reprogramming. Stem Cells, 2012, 30: 1405-1413

106 Vierbuchen T, Wernig M. Direct lineage conversions: unnatural but useful? Nat Biotechnol, 2011, 29: 892-907

107 Zhou Q, Brown J, Kanarek A, et al. In vivo reprogramming of adult pancreatic exocrine cells to beta-cells. Nature, 2008, 455: 627-632

108 Vierbuchen T, Ostermeier A, Pang Z P, et al. Direct conversion of fibroblasts to functional neurons by defined factors. Nature, 2010, 463: 1035-1041

109 Marro S, Pang Z P P, Yang N, et al. Direct lineage conversion of terminally differentiated hepatocytes to functional neurons. Cell Stem Cell, 2011, 9: 374-382

110 Ieda M, Fu J D, Delgado-Olguin P, et al. Direct reprogramming of fibroblasts into functional cardiomyocytes by defined factors. Cell, 2010, 142: 375-386

111 Song K, Nam Y J, Luo X, et al. Heart repair by reprogramming non-myocytes with cardiac transcription factors. Nature, 2012, 485: 599-604

112 Qian L, Huang Y, Spencer C I, et al. In vivo reprogramming of murine cardiac fibroblasts into induced cardiomyocytes. Nature, 2012, 485: 593-598

113 Huang $\mathrm{P} \mathrm{Y}$, He Z Y, Ji S Y, et al. Induction of functional hepatocyte-like cells from mouse fibroblasts by defined factors. Nature, 2011, 475: 386-389

114 Sekiya S, Suzuki A. Direct conversion of mouse fibroblasts to hepatocyte-like cells by defined factors. Nature, 2011, 475: 390393

115 Morris S A, Daley G Q. A blueprint for engineering cell fate: Current technologies to reprogram cell identity. Cell Res, 2013, 23: $33-48$
116 Yoo A S, Sun A X, Li L, et al. microRNA-mediated conversion of human fibroblasts to neurons. Nature, 2011, 476: 228-231

117 Jayawardena T M, Egemnazarov B, Finch E A, et al. microRNAmediated in vitro and in vivo direct reprogramming of cardiac fibroblasts to cardiomyocytes. Circ Res, 2012, 110: 1465-1473

118 Yoo A S, Staahl B T, Chen L, et al. microRNA-mediated switching of chromatin-remodelling complexes in neural development. Nature, 2009, 460: 642-646

119 Ambasudhan R, Talantova M, Coleman R, et al. Direct reprogramming of adult human fibroblasts to functional neurons under defined conditions. Cell Stem Cell, 2011, 9: 113-118

120 Nam Y J, Song K H, Luo X, et al. Reprogramming of human fibroblasts toward a cardiac fate. Proc Natl Acad Sci USA, 2013, 110: 5588-5593

121 Xue Y C, Ouyang K F, Huang J, et al. Direct conversion of fibroblasts to neurons by reprogramming PTB-regulated microRNA circuits. Cell, 2013, 152: 82-96

122 Lim L P, Lau N C, Garrett-Engele P, et al. Microarray analysis shows that some microRNAs downregulate large numbers of target mRNAs. Nature, 2005, 433: 769-773

123 Huang $\mathrm{M} \mathrm{B}, \mathrm{Xu} \mathrm{H}$, Xie S J, et al. Insulin-like growth factor-1 receptor is regulated by microRNA-133 during skeletal myogenesis. PLoS ONE, 2011, 6: e29173

124 Zhou A D, Diao L T, Xu H, et al. $\beta$-Catenin/LEF1 transactivates the microRNA-371-373 cluster that modulates the Wnt/beta-cateninsignaling pathway. Oncogene, 2012, 31: 2968-2978

125 Buganim Y, Faddah D A, Jaenisch R. Mechanisms and models of somatic cell reprogramming. Nat Rev Genet, 2013, 14: 427-439

126 Chi S W, Zang J B, Mele A, et al. Argonaute HITS-CLIP decodes microRNA-mRNA interaction maps. Nature, 2009, 460: 479-486

127 Hafner M, Landthaler M, Burger L, et al. Transcriptome-wide identification of RNA-binding protein and microRNA target sites by PAR-CLIP. Cell, 2010, 141: 129-141

128 Yang J H, Li J H, Shao P, et al. starBase: A database for exploring microRNA-mRNA interaction maps from Argonaute CLIP-Seq and Degradome-Seq data. Nucleic Acids Res, 2011, 39: D202-D209

129 Leung A K L, Young A G, Bhutkar A, et al. Genome-wide identification of Ago2 binding sites from mouse embryonic stem cells with and without mature microRNAs. Nat Struct Mol Biol, 2011, 18: 237-244

130 Lipchina I, Elkabetz Y, Hafner M, et al. Genome-wide identification of microRNA targets in human ES cells reveals a role for miR-302 in modulating BMP response. Gene Dev, 2011, 25: 2173-2186

131 Waddington $\mathrm{C} \mathrm{H}$. The Strategy of the Genes: A Discussion of Some Aspects of Theoretical Biology. London: George Allen \& Unwin, 1957

Open Access This article is distributed under the terms of the Creative Commons Attribution License which permits any use, distribution, and reproduction in any medium, provided the original author(s) and source are credited. 